\title{
Squeezing, Sub-Poissonian and Total Noise in Degenerate Six-Wave Mixing Process
}

\author{
B. Kumar Choudhary ${ }^{a, *}$ And D.K. Giri ${ }^{b}$ \\ ${ }^{a}$ Department of Applied Physics, Cambridge Institute of Technology, Tatisilwai, Ranchi, India \\ ${ }^{b}$ Department of Physics, P.K. Roy Memorial College, Dhanbad, India
}

(Received July 12, 2017; in final form July 25, 2018)

\begin{abstract}
We study a single-mode squeezing, sub-Poissonian and total noise in degenerate six-wave mixing process. We establish the analytic expression of first-order and second-order squeezing in terms of total noise under short-time approximation in degenerate six-wave mixing process. It shows that higher-order squeezing allows a much larger fractional noise reduction than lower-order squeezing. We observe that the squeezed states are associated with large number of photons. We find that squeezing is greater in stimulated process than corresponding squeezing in spontaneous interaction. The photon statistics of the pump mode in the process is investigated and found to be sub-Poissonian in nature. The effect of sub-Poissonian nature of an optical field in terms of total noise is also incorporated. We show that the depth of nonclassicality directly depends on the amount of total noise present in the system. This suggests that the more squeezed the state is, the greater is its total noise in the system.
\end{abstract}

DOI: 10.12693/APhysPolA.134.1108

PACS/topics: nonclassical state, higher order squeezing, photon number, total noise

\section{Introduction}

The "squeezing", "squeezed states", and "squeezed operator" were introduced by Hollenhorst and Caves [1, 2], but they were accepted after Walls' paper [3]. The first successful experiments on the generation and detection of squeezed states were reported in the middle of the 1980s [4-6]. Over the past decades, the squeezing [7-11] in quantized electromagnetic fields has received a great deal of attention because of its wide applications in many branches of science and technology, especially for low noise property [12-14] with an application in high quality telecommunication [15], quantum cryptography [16, 17], and so forth. A detailed review of squeezed states was presented by Dodonov et al. [18] and Anderson et al. [19]. The basic concept of squeezed light is concerned with the reduction of quantum fluctuations in one of the quadrature, at the expense of increased fluctuations in the other quadrature.

Squeezing has been focused on theoretical investigations and experimental observations in a variety of nonlinear optical processes, such as harmonic generation $[20,21]$, multiwave mixing processes [22-25], Raman [26-28], hyper-Raman [29], Hong and Mandel [30, 31], Hillery [32-34], and Zhan [35] for improving the performance of many optical devices and optical communication networks. Squeezing and photon statistical effect of the field amplitude in optical parametric, and in Raman and hyper Raman scattering processes has also been reported by Perina [36]. Higherorder sub-Poissonian photon statistics of light have also been studied by Kim and Yoon [37]. Recently, Prakash

*corresponding author; e-mail: binodvlsi@gmail.com and Mishra [38, 39] have reported the higher-order subPoissonian photon statistics and their use in detection higher-order squeezing. A proposal for experimental detection of amplitude $n$-th-power squeezing has been given by Prakash and Yadav [40]. The concept of total noise of a quantum state was introduced by Schumaker [41] in pure states and pointed out that the total noise is always greater than or equal to a half and reaches this value only for coherent state. Further, the notion of total noise in relation to squeezing of a field state has been given by Hillery [42] that reported about the measurement of the size of the field amplitude fluctuations of a state of the field. Furthermore, Gupta et al. [43] and Gill et al. [44] have also stated that the squeezing and total noise present in the system can be tuned by varying the phase angle.

The paper is organized for studying the concept of squeezing, sub-Poissonian and total noise in degenerate six-wave mixing (DSWM) process as follows. Section 2 gives definition of squeezing, sub-Poissonian and total noise of a quantum field state. We establish the analytic expression of spontaneous and stimulated first-order and second-order squeezing in DSWM process under shorttime approximation in Sect. 3. The effect of higher-order squeezing and sub-Poissonian nature of an optical field in terms of total noise are also incorporated in this section. Finally, we conclude this paper in Sect. 4 .

\section{Definition of squeezing, sub-Poissonian and total noise of a quantum field state}

The notion of total noise of quantum state of a single mode whose density matrix is $\rho$ can be defined [42] in terms of the operators

$$
X_{1}=\frac{1}{2}\left(A+A^{\dagger}\right) \quad \text { and } \quad X_{2}=\frac{1}{2 \mathrm{i}}\left(A-A^{\dagger}\right) .
$$


These operators correspond to the real and imaginary parts of the field amplitude, respectively, where $A \equiv A(t)$ and $A^{\dagger} \equiv A^{\dagger}(t)$ are the slowly varying operators because the interaction between modes, the operators $A(t)$ and $A^{\dagger}(t)$ induce a slower dependence on time as compared to fast variation of $a(t) \propto \exp (-\mathrm{i} \omega t)$ and $a^{\dagger}(t) \propto \exp (\mathrm{i} \omega t)$ that are useful in discussing squeezing effects. For a single mode of the electromagnetic field with frequency $\omega$ and creation (annihilation) operators $a^{\dagger},(a)$, they are given by $A(t)=a(t) \exp (\mathrm{i} \omega t)$ and $A^{\dagger}(t)=a^{\dagger}(t) \exp (-\mathrm{i} \omega t)$.

From Eq. (1), we find that

$$
\left\langle X_{1}^{2}\right\rangle+\left\langle X_{2}^{2}\right\rangle=\left\langle N+\frac{1}{2}\right\rangle \text {. }
$$

The total noise, which is measure of the total fluctuations of the amplitude, is

$$
T(\rho)=\left\langle\Delta X_{1}\right\rangle^{2}+\left\langle\Delta X_{2}\right\rangle^{2} .
$$

The uncertainty relation for $X_{1}$ and $X_{2}$ is

$$
\Delta X_{1} \Delta X_{2} \geq \frac{1}{4}
$$

which gives the condition for total noise as

$$
T(\rho) \geq\left\langle\Delta X_{1}\right\rangle^{2}+\frac{1}{\left\langle 4 \Delta X_{1}\right\rangle^{2}}
$$

Amplitude-squared squeezing [32-34] is defined as in terms of operators $Y_{1}$ and $Y_{2}$ as

$$
\begin{aligned}
& Y_{1}=\frac{1}{2}\left(A^{2}+A^{\dagger 2}\right) \\
& \text { and } Y_{1}=\frac{1}{2 \mathrm{i}}\left(A^{2}-A^{\dagger 2}\right),
\end{aligned}
$$

where $Y_{1}$ and $Y_{2}$ are the real and imaginary parts of the square of field amplitude, respectively. tion

The operators $Y_{1}$ and $Y_{2}$ obey the commutation rela-

$$
\left[Y_{1}, Y_{2}\right]=\mathrm{i}\left(N+\frac{1}{2}\right) .
$$

This leads to the uncertainty relation as

$$
\Delta Y_{1} \Delta Y_{2}=\left\langle N+\frac{1}{2}\right\rangle,
$$

where $N$ is the number operator.

Amplitude--squared squeezing is said to exist in $Y_{1}$ variable if

$$
\left(\Delta Y_{1}\right)^{2}<\left\langle N+\frac{1}{2}\right\rangle
$$

Increased nonclassicality gives rise to increase in the total noise $[42,43]$. This fact can be verified by associating total noise with higher-order squeezing.

In order to relate the total noise to the uncertainty relations for the quadrature variables, using Hillery's approach [42] may be as follows:

$$
\begin{aligned}
& \left(\Delta X_{1}\right)^{2}\left(\Delta Y_{1}\right)^{2} \geq \frac{1}{4}\left\langle X_{2}\right\rangle^{2}, \\
& \left(\Delta X_{2}\right)^{2}\left(\Delta Y_{1}\right)^{2} \geq \frac{1}{4}\left\langle X_{1}\right\rangle^{2} .
\end{aligned}
$$

If above these two inequalities combined with Eq. (2), we find

$$
\begin{aligned}
& 4\left(\Delta Y_{1}\right)^{2}\left[\left(\Delta X_{1}\right)^{2}+\left(\Delta X_{2}\right)^{2}\right] \geq \\
& \left\langle N+\frac{1}{2}\right\rangle-\left[\left(\Delta X_{1}\right)^{2}+\left(\Delta X_{2}\right)^{2}\right] .
\end{aligned}
$$

On simplification, we get

$$
T_{N}=\left(\Delta X_{1}\right)^{2}+\left(\Delta X_{2}\right)^{2} \geq \frac{\left\langle N+\frac{1}{2}\right\rangle}{\left[4\left(\Delta Y_{1}\right)^{2}+1\right]} .
$$

We see that, for fixed $\langle N\rangle$ as $\Delta Y_{1}$ decreases, the total noise must increase. $T_{N}$ increases as a state becomes more squeezed and may be considered as a measure of depth of nonclassicality.

A state is sub-Poissonian if $(\Delta N)^{2}<\langle N\rangle$ where $N=$ $A^{\dagger} A$ and $(\Delta N)^{2}=\left\langle(N-\langle N\rangle)^{2}\right\rangle$. In order to relate number operator to the total noise of quantum state, let us use the Schwartz inequality,

$$
\begin{aligned}
& \left|\left\langle\left(X_{1}-\left\langle X_{1}\right\rangle\right)(N-\langle N\rangle)\right\rangle\right|^{2} \leq \\
& \quad\left\langle\left(X_{1}-\left\langle X_{1}\right\rangle\right)^{2}\right\rangle\left\langle(N-\langle N\rangle)^{2}\right\rangle \leq\left(\Delta X_{1}\right)^{2}(\Delta N)^{2} .
\end{aligned}
$$

This implies that

$$
\begin{aligned}
\left(\Delta X_{1}\right)^{2}(\Delta N)^{2} & \geq\left|\left\langle\left(X_{1}-\left\langle X_{1}\right\rangle\right)(N-\langle N\rangle)\right\rangle\right|^{2} \geq \\
\frac{1}{4}\left|\left\langle\left[X_{1}, N\right]\right\rangle\right|^{2} & \geq \frac{1}{4}\left|\left\langle X_{2}\right\rangle\right|^{2},
\end{aligned}
$$

where $\left[X_{1}, N\right]=\mathrm{i} X_{2}$.

Similarly

$$
\left(\Delta X_{2}\right)^{2}(\Delta N)^{2} \geq \frac{1}{4}\left|\left\langle X_{1}\right\rangle\right|^{2} .
$$

From Eqs. (14) and (15), we have

$$
4(\Delta N)^{2}\left[\left(\Delta X_{1}\right)^{2}+\left(\Delta X_{2}\right)^{2}\right] \geq\left\langle X_{1}\right\rangle^{2}+\left\langle X_{2}\right\rangle^{2} .
$$

Using Eq. (2) and simplifying, we get

$$
\left[4(\Delta N)^{2}+1\right]\left[\left(\Delta X_{1}\right)^{2}+\left(\Delta X_{2}\right)^{2}\right] \geq\left\langle N+\frac{1}{2}\right\rangle \text {. }
$$

Therefore, the total noise in quantum state in terms of number operator is

$$
T_{N}=\left[\left(\Delta X_{1}\right)^{2}+\left(\Delta X_{2}\right)^{2}\right] \geq \frac{\left\langle N+\frac{1}{2}\right\rangle}{\left[4(\Delta N)^{2}+1\right]} .
$$

From Eq. (18) it is clear that when $(\Delta N)^{2}$ decreases, the total noise must increase.

\section{Squeezing, sub-Poissonian and total noise in fundamental mode in DSWM process}

In this model, the process involving absorption of three pump photons of frequency $\omega_{1}$ each, going from state $|1\rangle$ to state $|2\rangle$ and emission of two probe photons from state $|2\rangle$ to state $|3\rangle$ with frequency $\omega_{2}$ each. The atomic system returns to its original state by emitting one signal photon of frequency $\omega_{3}$ from $|3\rangle$ to $|1\rangle$ as shown in Fig. 1 .

The Hamiltonian for this process is as follows $(\hbar=1)$ :

$H=\omega_{1} a^{\dagger} a+\omega_{2} b^{\dagger} b+\omega_{3} c^{\dagger} c+g\left(a^{3} b^{\dagger 2} c^{\dagger}+a^{\dagger 3} b^{2} c\right)$,

where $a^{\dagger}(a), b^{\dagger}(b)$ and $c^{\dagger}(c)$ are the creation (annihilation) operators of the $A, B$, and $C$ modes, respectively, 


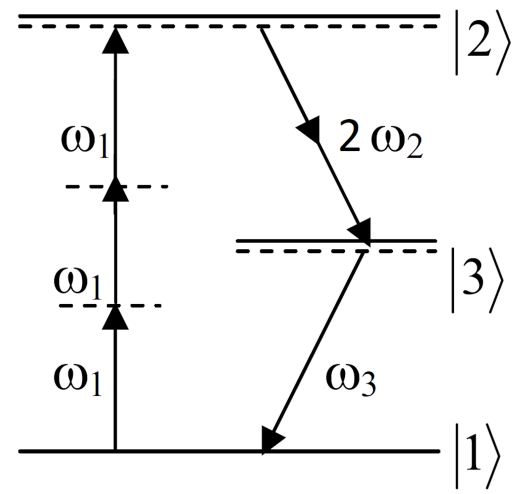

Fig. 1. Degenerate six-wave interaction model [45].

and $g$ is the coupling constant between the two modes per second. $A, B$, and $C$ are slowly varying operators for the three modes at $\omega_{1}, \omega_{2}$, and $\omega_{3}$, which are defined by $A=a \exp \left(\mathrm{i} \omega_{1} t\right), B=b \exp \left(\mathrm{i} \omega_{2} t\right)$ and $C=c \exp \left(\mathrm{i} \omega_{3} t\right)$, associated with the relation $3 \omega_{1}=2 \omega_{2}+\omega_{3}$.

The Heisenberg equation of motion for mode $A$ is

$$
\dot{A}=\frac{\partial A}{\partial t}+\mathrm{i}[H, A] \text {. }
$$

Using (19) in (20), we obtain

$$
\dot{A}=-3 \mathrm{i} g A^{\dagger 2} B^{2} C \text {. }
$$

Similarly, we obtain the relations for $\dot{B}$ and $\dot{C}$ as

$$
\dot{B}=-2 \mathrm{i} g A^{3} B^{\dagger} C^{\dagger} \text { and } \dot{C}=-\mathrm{i} g A^{3} B^{\dagger 2} \text {. }
$$

Expanding $A(t)$ using Taylor's series expansion by assuming the short-interaction of wave with the medium and retaining up to $|g t|^{2}$, we obtain

$$
\begin{gathered}
\dot{A}(t)=A-3 \mathrm{i} g t A^{\dagger 2} B^{2} C+\frac{3}{2} g^{2} t^{2}\left(6 A^{\dagger} A^{2} B^{\dagger 2} B^{2} C^{\dagger} C\right. \\
+6 A B^{\dagger 2} B^{2} C^{\dagger} C-4 A^{\dagger 2} A^{3} B^{\dagger} B C^{\dagger} C-2 A^{\dagger 2} A^{3} C^{\dagger} C \\
\left.-A^{\dagger 2} A^{3} B^{\dagger 2} B^{2}-4 A^{\dagger 2} A^{3} B^{\dagger} B-2 A^{\dagger 2} A^{3}\right) .
\end{gathered}
$$

The real quadrature component for squeezing of field amplitude in fundamental mode $A$ is given as

$$
X_{1 A}(t)=\frac{1}{2}\left[A(t)+A^{\dagger}(t)\right]
$$

For spontaneous interaction, we consider the quantum state as a product of coherent state for the fundamental mode $A$ and the vacuum state for the mode $B$ and $C$, that is

$$
|\psi\rangle=|\alpha\rangle_{A}|0\rangle_{B}|0\rangle_{C},
$$

where $\alpha$ is the complex amplitude of the fundamental mode. Using (23)-(25), we obtain the expectation value as

$$
\begin{aligned}
& \left\langle\psi\left|X_{1 A}^{2}(t)\right| \psi\right\rangle=\frac{1}{4}\left[\alpha^{2}+\alpha^{* 2}+2|\alpha|^{2}+1-6 g^{2} t^{2}\left(\alpha^{2}|\alpha|^{4}\right.\right. \\
& \left.+\alpha^{2}|\alpha|^{2}+\alpha^{* 2}|\alpha|^{4}+\alpha^{* 2}|\alpha|^{2}+2|\alpha|^{6}\right], \\
& \left\langle\psi\left|X_{1 A}(t)\right| \psi\right\rangle^{2}=\frac{1}{4}\left[\alpha^{2}+\alpha^{* 2}+2|\alpha|^{2}-6 g^{2} t^{2}\left(\alpha^{2}|\alpha|^{4}\right.\right. \\
& \left.+\alpha^{* 2}|\alpha|^{4}+2|\alpha|^{6}\right] .
\end{aligned}
$$

Therefore,

$$
\begin{gathered}
{\left[\Delta X_{1 A}(t)\right]^{2}=\left\langle X_{1 A}^{2}(t)\right\rangle-\left\langle X_{1 A}(t)\right\rangle^{2}=} \\
\frac{1}{4}\left[1-6 g^{2} t^{2}\left(\alpha^{2}|\alpha|^{2}+\alpha^{* 2}|\alpha|^{2}\right)\right], \\
{\left[\Delta X_{1 A}(t)\right]^{2}-\frac{1}{4}=-3 g^{2} t^{2}|\alpha|^{4} \cos 2 \theta,}
\end{gathered}
$$

where $\theta$ is the phase angle, with $\alpha=|\alpha| \mathrm{e}^{\mathrm{i} \theta}$ and $\alpha^{*}=$ $|\alpha| \mathrm{e}^{-\mathrm{i} \theta}$.

The right hand side of the expression (29) is negative, indicating that squeezing will occur in the first-order amplitude in the fundamental mode in degenerate six-wave mixing process for which $\cos 2 \theta>0$ for spontaneous interaction.

Spontaneous emission is an effect caused by the coupling of the atom to the vacuum state. Analogously, stimulated emission is caused by the coupling of the atom to the other states of the field. To study squeezing in stimulated interaction in DSWM process we assume an initial quantum state as a product of coherent states $|\alpha\rangle$ for the fundamental mode $A,|\beta\rangle$ for the Stokes mode $B$ and vacuum state $|0\rangle$ for the mode $C$,

$$
|\psi\rangle=|\alpha\rangle_{A}|\beta\rangle_{B}|0\rangle_{C} \text {. }
$$

We obtain expectation value as

$$
\begin{aligned}
& \left\langle\psi\left|X_{1 A}(t)\right| \psi\right\rangle^{2}=\frac{1}{4}\left[\alpha^{2}+\alpha^{* 2}+2|\alpha|^{2}-3 g^{2} t^{2}\right. \\
& \left.\times\left(\alpha^{2}|\alpha|^{4}+\alpha^{* 2}|\alpha|^{4}+2|\alpha|^{6}\right) \times\left(|\beta|^{4}+4|\beta|^{2}+2\right)\right], \\
& \left\langle\psi\left|X_{1 A}^{2}(t)\right| \psi\right\rangle=\frac{1}{4}\left[\alpha^{2}+\alpha^{* 2}+2|\alpha|^{2}+1-3 g^{2} t^{2}\right. \\
& \times\left(\alpha^{2}|\alpha|^{4}+\alpha^{2}|\alpha|^{2}+\alpha^{* 2}|\alpha|^{4}+\alpha^{* 2}|\alpha|^{2}+2|\alpha|^{6}\right) \\
& \left.\quad \times\left(|\beta|^{4}+4|\beta|^{2}+2\right)\right] .
\end{aligned}
$$

Therefore,

$$
\begin{aligned}
& {\left[\Delta X_{1 A}(t)\right]^{2}-\frac{1}{4}=} \\
& \quad-\frac{3}{2} g^{2} t^{2}|\alpha|^{4}\left(|\beta|^{4}+4|\beta|^{2}+2\right) \cos 2 \theta .
\end{aligned}
$$

The right hand side of Eq. (33) is always negative, showing the existence of squeezing in the first-order amplitude in the fundamental mode in stimulated DSWM process for those value of $\theta$ for which $\cos 2 \theta>0$. The factor $\left(|\beta|^{4}+4|\beta|^{2}+2\right)$ in Eq. (33) is the effect of stimulated interaction.

The results show the presence of squeezing in fundamental mode in DSWM process in the first-order. To study normal (first-order) squeezing, we denote the right hand side of Eq. (33) by $S_{x}$. Taking $|g t|^{2}=10^{-8}$ and $\theta=0$, the variations of $S_{x}$ is shown in Fig. 2. We have used $\theta=0$ to get maximum squeezing.

The steady fall of the curve shows an increase in the degree of squeezing with number of photons. Figure 2 shows that the squeezing increases nonlinearly with $|\alpha|^{2}$ which is directly dependent upon the number of photons. 


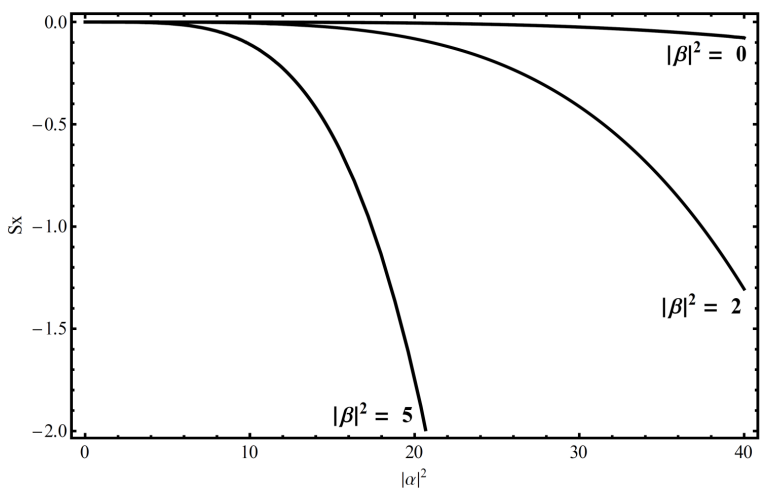

Fig. 2. Variation of the first-order spontaneous $\left(|\beta|^{2}=\right.$ 0 ) and stimulated squeezing $S_{x}$ with $|\alpha|^{2}$ (when $|g t|^{2}=$ $10^{-8}$ and $\left.\theta=0\right)$.

Further, in stimulated process squeezing also increases with $|\beta|^{2}$ that is the photon number in $B$ mode. This confirms that the squeezed states are associated with large number of photons.

Further the total noise in this process can be measured as

$$
\begin{aligned}
& \left(T_{N}\right)_{x} \geq\left[\Delta X_{1 A}(t)\right]^{2}+\frac{1}{4\left[\Delta X_{1 A}(t)\right]^{2}} \geq \\
& \frac{1}{4}-3 g^{2} t^{2}|\alpha|^{4} \cos 2 \theta+\frac{1}{1-12 g^{2} t^{2}|\alpha|^{4} \cos 2 \theta} .
\end{aligned}
$$

The expression on the left-hand side of the above inequality reaches a minimum when $\Delta X_{1}=\frac{1}{2}$. The value of $\Delta X_{1}$ indicates the classical region. For $0<\Delta X_{1}<\frac{1}{2}$, as $\Delta X_{1}$ decreases $T_{N}$ increases.

The variation of first-order squeezing in terms of total noise with $|\alpha|^{2}$ is shown in Fig. 3 .

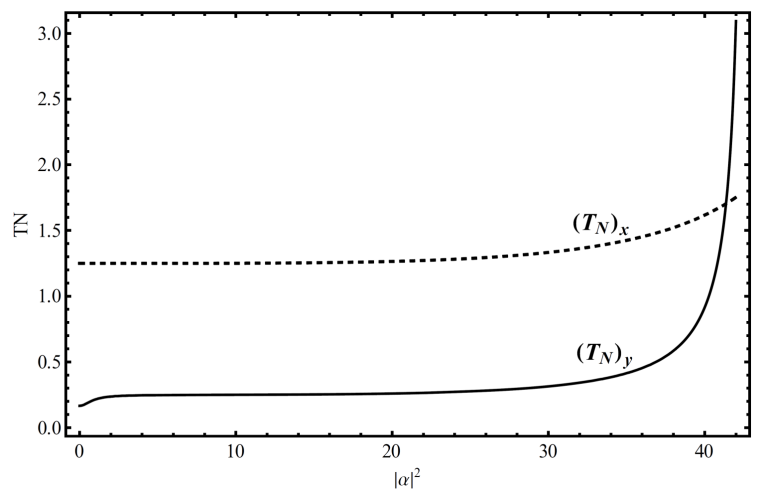

Fig. 3. Variation of total noise $\left(T_{N}\right)$ in quantum state with $|\alpha|^{2}$ (when $|g t|^{2}=10^{-8}$ and $\theta=0$ ) (dashed line: first-order $\left(T_{N}\right)_{x}$ and solid line: second-order $\left.\left(T_{N}\right)_{y}\right)$.

Using (23) and (25), the second order amplitude is expressed as

$$
\begin{aligned}
& A^{2}(t)=A^{2}-6 g^{2} t^{2}\left(A^{\dagger 2} A^{4}+A^{\dagger} A^{3}\right) \\
& \text { and } A^{\dagger 2}(t)=A^{\dagger 2}-6 g^{2} t^{2}\left(A^{\dagger 4} A^{2}+A^{\dagger 3} A\right) .
\end{aligned}
$$

For second-order squeezing, the real expectation values for the fundamental mode is expressed as

$$
Y_{1 A}(t)=\frac{1}{2}\left[A^{2}(t)+A^{\dagger 2}(t)\right]
$$

Using (25) and (35) in (36), we get the expectation value in spontaneous degenerate six-wave mixing process as

$$
\begin{gathered}
\left\langle\psi\left|Y_{1 A}(t)\right| \psi\right\rangle^{2}=\frac{1}{4}\left[\alpha^{4}+\alpha^{* 4}+2|\alpha|^{4}-12 g^{2} t^{2}\left(\alpha^{4}|\alpha|^{4}\right.\right. \\
\left.+\alpha^{4}|\alpha|^{2}+\alpha^{* 4}|\alpha|^{4}+\alpha^{* 4}|\alpha|^{2}+2|\alpha|^{8}+2|\alpha|^{6}\right] \\
\left\langle\psi\left|Y_{1 A}^{2}(t)\right| \psi\right\rangle=\frac{1}{4}\left[\alpha^{4}+\alpha^{* 4}+2|\alpha|^{4}+4|\alpha|^{2}+2\right. \\
-12 g^{2} t^{2}\left(\alpha^{4}|\alpha|^{4}+3 \alpha^{4}|\alpha|^{2}+2 \alpha^{4}+\alpha^{* 4}|\alpha|^{4}\right. \\
\left.+3 \alpha^{* 4}|\alpha|^{2}+2 \alpha^{* 4}+2|\alpha|^{8}+10|\alpha|^{6}+18|\alpha|^{4}+6|\alpha|^{2}\right] .
\end{gathered}
$$

Therefore,

$$
\begin{aligned}
& {\left[\Delta Y_{1 A}(t)\right]^{2}=\left\langle Y_{1 A}^{2}(t)\right\rangle-\left\langle Y_{1 A}(t)\right\rangle^{2}=} \\
& \quad \frac{1}{4}\left[4|\alpha|^{2}+2-24 g^{2} t^{2}\left(\alpha^{4}|\alpha|^{2}+\alpha^{4}+\alpha^{* 4}|\alpha|^{2}+\alpha^{* 4}\right.\right. \\
& \left.\quad+4|\alpha|^{6}+9|\alpha|^{4}+3|\alpha|^{2}\right] .
\end{aligned}
$$

Using Eq. (25) and then the number of photon in mode $A$ may be expressed as

$$
N_{1 A}(t)=A^{\dagger}(t) A(t)=A^{\dagger} A-6 g^{2} t^{2} A^{\dagger 3} A^{3} .
$$

Then, we have

$$
\left\langle N_{1 A}(t)+\frac{1}{2}\right\rangle=\left[|\alpha|^{2}+\frac{1}{2}-6 g^{2} t^{2}|\alpha|^{6}\right] .
$$

Subtracting (41) from (39), we get

$$
\begin{aligned}
& {\left[\Delta Y_{1 A}(t)\right]^{2}-\left\langle N_{1 A}(t)+\frac{1}{2}\right\rangle=} \\
& \quad-6 g^{2} t^{2}\left[2 \cos 4 \theta\left(|\alpha|^{4}+|\alpha|^{6}\right)+3|\alpha|^{6}+9|\alpha|^{4}+3|\alpha|^{2}\right] .
\end{aligned}
$$

Using initial condition (30), we obtain squeezing for the stimulated process as

$$
\begin{aligned}
& {\left[\Delta Y_{1 A}(t)\right]^{2}-\left\langle N_{1 A}(t)+\frac{1}{2}\right\rangle=-3 g^{2} t^{2}\left[2 \cos 4 \theta\left(|\alpha|^{4}+|\alpha|^{6}\right)\right.} \\
& \left.+3|\alpha|^{6}+9|\alpha|^{4}+3|\alpha|^{2}\right]\left(|\beta|^{4}+4|\beta|^{2}+2\right) .
\end{aligned}
$$

The right hand side of Eqs. (42) and (43) are negative for all values of $\theta$ for which $\cos 4 \theta>0$ and thus shows the existence of squeezing in the second order of the field amplitude in spontaneous and stimulated under shorttime approximation. The factor $\left(|\beta|^{4}+4|\beta|^{2}+2\right)$ in Eq. (43) is the effect of stimulated interaction.

The results show the presence of squeezing in fundamental mode in DSWM process in second-order. To study higher-order (second-order) squeezing, we denote the right hand side of Eq. (43) by $S_{y}$. Taking $|g t|^{2}=10^{-8}$ and $\theta=0$, the variations of $S_{y}$ is shown in Fig. 4 .

It is clear from the plot that second-order squeezing increases nonlinearly with $|\alpha|^{2}$. Thus, the degree of squeezing is directly dependent on the photon number in fundamental mode. Further, in stimulated case squeezing also increases with $|\beta|^{2}$, that is the photon number in $B$ mode. 


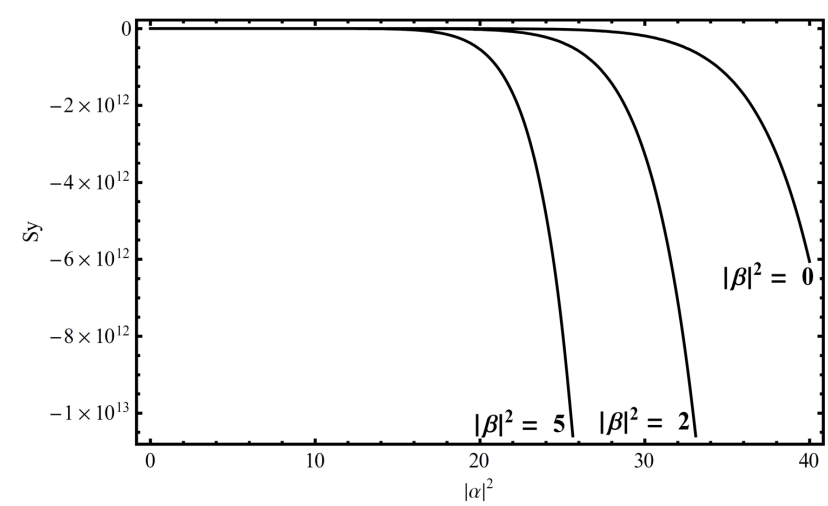

Fig. 4. Variation of the second-order spontaneous $\left(|\beta|^{2}=0\right.$ ) and stimulated squeezing $S_{y}$ with $|\alpha|^{2}$ (when $|g t|^{2}=10^{-8}$ and $\left.\theta=0\right)$.

A comparison between Figs. 2 and 4 shows greater noise reduction in second-order than in first-order, having same number of photons.

A comparison between results for spontaneous and stimulated process shows the occurrence of a multiplication factor $\left(|\beta|^{4}+4|\beta|^{2}+2\right)$, which tells that squeezing in the fundamental mode in stimulated interaction is greater than corresponding squeezing in spontaneous interaction and also shows that maximum squeezing will occur when only $\theta \rightarrow 0$ and minimum squeezing for $\theta \rightarrow \pi / 2$ and $\theta \rightarrow \pi / 4$, respectively, for first and second-order.

Further the total noise in terms of second-order squeezing,

$$
\begin{aligned}
\left(T_{N}\right)_{y} & \geq \frac{\left\langle N_{1 A}(t)+\frac{1}{2}\right\rangle}{4\left[\Delta Y_{1 A}(t)\right]^{2}+1} \geq|\alpha|^{2}+\frac{1}{2}-6 g^{2} t^{2}|\alpha|^{6} \\
& \times\left\{4|\alpha|^{2}+3-24 g^{2} t^{2}\left[\left(|\alpha|^{4}+|\alpha|^{6}\right) 2 \cos 4 \theta\right.\right. \\
& \left.\left.+4|\alpha|^{6}+9|\alpha|^{4}+3|\alpha|^{2}\right]\right\}^{-1} .
\end{aligned}
$$

From Eq. (44), we see that for fixed $\langle N\rangle$ as $[\Delta Y(t)]^{2}$ decreases, the total noise must increase.

The variation of second-order squeezing in terms of total noise with $|\alpha|^{2}$ is shown in Fig. 3. For $|\beta|^{2}=0$ corresponds to spontaneous process. The steady increase of the curves (Fig. 3) shows that the total noise increases with increase of the number of photons $\left(|\alpha|^{2}\right)$. It infers that the depth of nonclassicality directly depends on the large number of photons. This also suggests that the more squeezed the state is, the greater is its total noise.

A comparison between figures shows greater total noise and hence greater squeezing in second-order than in firstorder, having the same number of photons. The result agrees with the result of Hillery [42] that the maximum total noise is possible in higher-order (second-order) squeezing. It again infer that the more squeezed the state is, the greater is its total noise.

Using (25) and (40), the statistics of fundamental mode in degenerate six-wave mixing is found to be subPoissonian, as

$$
\left[\Delta N_{1 A}(t)\right]^{2}-\left\langle N_{1 A}(t)\right\rangle=-30 g^{2} t^{2}|\alpha|^{6} .
$$

For studying sub-Poissonian photon statistics nature in nonclassical state, let us denote the right-hand side of Eq. (45) by $S_{n}$. Taking $|g t|^{2}=10^{-8}$, the variations of $S_{n}$ with $|\alpha|^{2}$ is shown in Fig. 5 .

Figure 5 shows that the sub-Poissonian statistics properties of light is directly proportional to the number of photons in fundamental mode $A$ i.e. sub-Poissonian statistics of light increases with increase of $|\alpha|^{2}$. Thus, the degree of sub-Poissonian photon statistics is also associated with large number of photons.

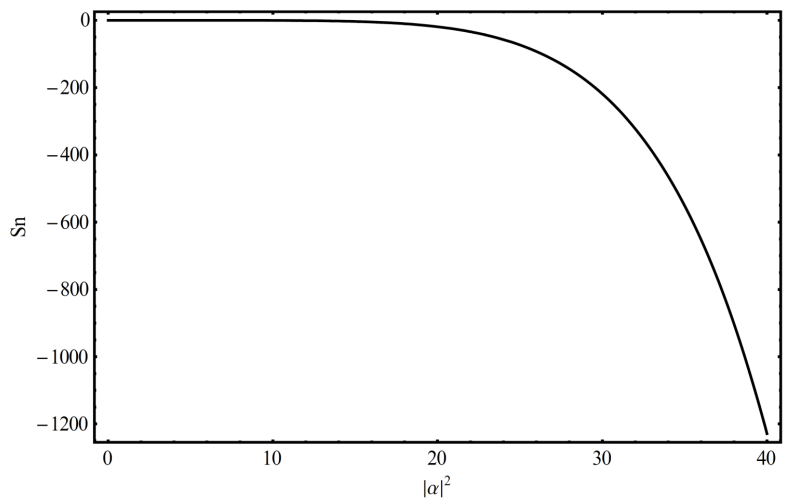

Fig. 5. Variation of sub-Poissonian states $\left(S_{n}\right.$ with $|\alpha|^{2}$ (when $|g t|^{2}=10^{-8}$ and $\theta=0$ ).

Hence, the total noise related to number operator

$$
\begin{gathered}
\left(T_{N}\right)_{n} \geq \frac{\left\langle N_{1 A}(t)+\frac{1}{2}\right\rangle}{4\left[\Delta N_{1 A}(t)\right]^{2}+1} \geq \\
\frac{|\alpha|^{2}+\frac{1}{2}-6 g^{2} t^{2}|\alpha|^{6}}{4|\alpha|^{2}+1-144 g^{2} t^{2}|\alpha|^{6}} .
\end{gathered}
$$

From above Eq. (46), it is evident that for fixed $\langle N\rangle$ as $[\Delta N(t)]^{2}$ decreases, then $T_{N}$ must increase. The variation of total noise in terms of number operator $\left(\mathrm{T}_{N}\right)_{n}$ with $|\alpha|^{2}$ is shown in Fig. 6. The

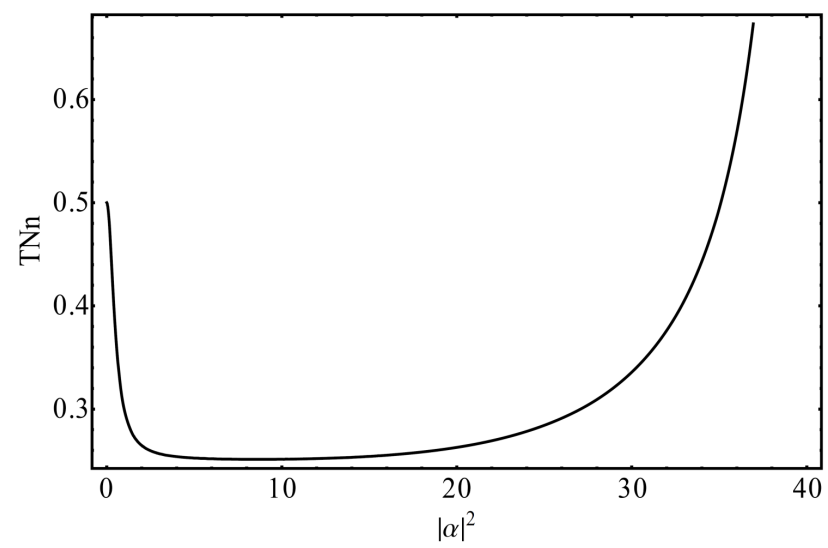

Fig. 6. Variation of total noise $\left(T_{N}\right)_{n}$ in subPoissonian state with $|\alpha|^{2}$ (when $|g t|^{2}=10^{-8}$ and $\theta=0)$. 
steady increase of the curves (Fig. 6) shows the total noise increases with increase of the number of photons $|\alpha|^{2}$. Therefore, as state becomes more sub-Poissonian $[\Delta N(t) /\langle N\rangle=$ decreasing $]$ its total noise increases.

\section{Conclusions}

The results show the presence of squeezing in fundamental mode in DSWM process in first-order as well as in second-order. A comparison between results of spontaneous and stimulated processes shows the occurrence of a multiplication factor $\left(|\beta|^{4}+4|\beta|^{2}+2\right)$, which tells that squeezing in the fundamental mode in stimulated process is greater than corresponding squeezing in spontaneous process and show that maximum squeezing will occur when $\theta \rightarrow 0$.

A comparison of squeezing in spontaneous interaction with that of stimulated in DSWM process shows larger degree of squeezing in the stimulated interaction for the same order. Thus it may be inferred that higher degree of squeezing is associated with higher degree of nonlinearity of the optical process. It also show that the squeezing increases nonlinearly with $|\alpha|^{2}$, which is directly dependent upon the number of photons. This confirms that the squeezed states are associated with large number of photons. Hence higher-order squeezing makes it possible to achieve significantly larger noise reduction than ordinary (or second-order) squeezing. This also establishes the fact that processes with higher-order nonlinearity is more suitable for generation of squeezed light.

It is found that the depth of nonclassicality is more in second order than first-order. Hence second-order will give more squeezed laser light than first-order.

On comparing curves in Fig. 3, it is concluded that greater total noise and hence greater squeezing exists in second-order than in first-order, having same number of photons. The result agrees with the result of Hillery [42]. Hence the maximum total noise is possible in higherorder (second-order) squeezing. It infers that the more squeezed the state is, the greater is its total noise in the system.

Figure 5 shows that the sub-Poissonian statistics properties of light is directly proportional to number of photons in the fundamental mode i.e. sub-Poissonian states of light increases with increase of $|\alpha|^{2}$. Thus, the degree of sub-Poissonian photon statistics is also associated with large number of photons.

Figure 6 shows that the total noise increases with increase of the number of photons $|\alpha|^{2}$. Therefore, as state becomes more sub-Poissonian, its total noise increases.

These results suggest that the desired degree of squeezing, sub-Poissonian and total noise can be obtained by using short interaction time and number of photons present in the radiation field before interaction in the system. Hence, the total noise of a quantum state can be measured the depth of nonclassicality i.e. more nonclassical a state (squeezing and sub-Poissonian) of the field in any system.

\section{Acknowledgments}

We would like to thank the referees for his comments and valuable suggestions.

\section{References}

[1] J.N. Hollenhorst, Phys. Rev. D 19, 1669 (1979).

[2] C.M. Caves, Phys. Rev. D 23, 1693 (1981).

[3] D.F. Walls, Nature 306, 141 (1983).

[4] R.E. Slusher, L.W. Hollberg, B. Yurke, J.C. Mertz, J.F. Valley, Phys. Rev. Lett. 55, 2409 (1985).

[5] R.M. Shelby, M.D. Levenson, S.H. Perlmutter, R.G. DeVoe, D.F. Walls, Phys. Rev. Lett. 57, 691 (1986).

[6] L.A. Wu, H.J. Kimble, J.L. Hall, H. Wu, Phys. Rev. Lett. 57, 2520 (1986).

[7] R. Loudon, P.L. Knight, J. Mod. Opt. 34, 709 (1987).

[8] M.C. Teich, B.E.A. Saleh, Quant. Opt. 1, 153 (1989).

[9] J. Perina, Quantum Statistics of Linear and Nonlinear Optical Phenomena, Kluwer, Dordrecht 1991, Chs. 9 and 10.

[10] L. Mandel, Phys. Scr. T 12, 34 (1986).

[11] V.V. Dodonov, J. Opt. B Quant. Semiclass. Opt. 4, R1 (2002).

[12] B.E.A. Saleh, M.C. Teich, Phys. Rev. Lett. 58, 2656 (1987).

[13] K. Wódkiewicz, J. Mod. Opt. 34, 941 (1987).

[14] H.J. Kimble, D.F. Walls, J. Opt. Soc. Am. B 4, 1450 (1987).

[15] H.P. Yuen, J.H. Shapiro, IEEE Trans. Inf. Theory 24, 657 (1978).

[16] C.H. Bennett, G. Brassard, N.D. Mermin, Phys. Rev.. Lett. 68, 557 (1992).

[17] J. Kempe, Phys. Rev. A 60, 910 (1999).

[18] V.V. Dodonov, M.A. Man'ko, V.I. Man'ko, A. Vourdas, J. Russ. Laser Res. 28, 404 (2007).

[19] U.L. Andersen, T. Gehring, C. Marquardt, G. Leuchs, Phys. Scr. 91, 053001 (2016).

[20] L. Mandel, Opt. Commun. 42, 437 (1982).

[21] S. Kielich, R. Tanas, R. Zawodny, J. Opt. Soc. Am. B 4, 1627 (1987).

[22] J. Perina, V. Perinova, C. Sibilia, M. Bertolotti, Opt. Commun. 49, 285 (1984).

[23] M.S.K. Razmi, J.H. Eberly, Opt. Commun. 76, 265 (1990).

[24] D.K. Giri, P.S. Gupta, J. Opt. B Quant. Semiclass. Opt. 6, 91 (2004).

[25] D.K. Giri, P.S. Gupta, Opt. Commun. 221, 135 (2003).

[26] J. Perina, J. Krepelka, J. Mod. Opt. 38, 2137 (1991).

[27] A. Kumar, P.S. Gupta, Quant. Semiclass. Opt. 7, 835 (1995).

[28] A. Kumar, P.S. Gupta, Quant. Semiclass. Opt. 8, 1053 (1996).

[29] D.K. Giri, P.S. Gupta, J. Mod. Opt. 52, 1769 (2005).

[30] C.K. Hong, L. Mandel, Phys. Rev. Lett. 54, 323 (1985). 
[31] C.K. Hong, L. Mandel, Phys. Rev. A 32, 974 (1985).

[32] M. Hillery, Opt. Commun. 62, 135 (1987).

[33] M. Hillery, Phys. Rev. A 36, 3796 (1987).

[34] M. Hillery, Phys. Rev. A 45, 4944 (1992).

[35] You-bang Zhan, Phys. Lett. A 160, 498 (1991).

[36] J. Perina, V. Perinova, J. Kodousek, Opt. Commun. 49, 210 (1984).

[37] Y. Kim, T.H. Yoon, Opt. Commun. 212, 107 (2002).

[38] H. Prakash, D.K. Mishra, J. Phys. B At. Mol. Opt. Phys. 39, 2291 (2006).

[39] D.K. Mishra, Opt. Commun. 283, 3284 (2010).
[40] R. Prakash, A.K. Yadav, Opt. Commun. 285, 2387 (2012).

[41] B.L. Schumaker, Phys. Rep. 135, 317 (1986).

[42] M. Hillery, Phys. Rev. A 39, 2994 (1989).

[43] P. Gupta, A. Pathak, Optik Int. J. Light Electron Opt. 121, 1507 (2010).

[44] S. Gill, Sunil Rani, Nafa Singh, Int. J. Opt. 2012, 1 (2012).

[45] Sunil Rani, Jawahar Lal, Nafa Singh, Int. J. Opt. 2011, 1 (2011). 